\title{
Study of thin substrates for microstrip gas chambers *
}

\author{
S. Schmidt, U. Werthenbach and G. Zech \\ Universität Siegen
}

July 19, 1993

\begin{abstract}
Microstrips have been produced on glass and plastic supports of various thickness. Flexible, $100 \mu \mathrm{m}$ thick glass plates were successfully operated. Glass foils are less vulnerable to sparks than plastic foils, are less sensitive to intensity changes and respond faster to voltage changes but have similar advantages. A structure on a $7 \mu m$ thin polyimide foil showed gas amplification with slightly reduced energy resolution as compared to the same structure on a thick foil. Its time stability during irradiation was unsatisfactory.
\end{abstract}

\section{Introduction}

In microstrip gas chambers (MSGC) the wires of the standard multiwire proportional chambers are replaced by lithographically produced strips on solid substrates. These devices have had many interesting developments since the first realization by A. Oed [1]. Several groups $[2,3,4]$ have built such devices. Experimental applications in high energy physics [5], synchrotron radiation [6] and heavy ion physics have been successful.

Besides glass, quartz and silicon [7] have been used as substrates. We have demonstrated the feasibility of microstrip chambers on 50 micrometer thick polyimide (Kapton $\mathrm{H}$, Dupont de Nemours Co.) foils [8]. Stable operation at moderate gas gains of about $10^{3}$ has been obtained. The usability of plastic supports in microstrip chambers was confirmed [9] and extended to larger areas and to another substrate, polyvinylfluoride (Tedlar, Dupont de Nemours Co.).

Thin, flexible substrates obviously offer distinct advantages in charged particle detection when compared to rigid supports: The substantially reduced amount of material minimizes multiple scattering and photon conversion and provides the possibility to readout induced signals at the back plane. They allow to realize cylindrical chambers with circular anode lines, which are potentially very interesting detectors in colliding beam experiments.

During our tests with plastic foil chambers we occasionally suffered from discharges, which destroyed the plastic supports. Bouclier et al. [9] observed variations in gain with time and irradiation intensity, which they attributed to polarization effects and charge up of the surface by deposition of positive ions, respectively. These defects are expected to be smaller or absent for thin flexible glass plates.

\footnotetext{
*Work supported by Bundesminister für Forschung und Technologie (FK 055Si77P)
} 



\begin{tabular}{|c|c|c|c|}
\hline Geometry & anode width & cathode width & gap anode-cath. \\
\hline I & $8 \mu \mathrm{m}$ & $200 \mu \mathrm{m}$ & $120 \mu \mathrm{m}$ \\
II & $10 \mu \mathrm{m}$ & $200 \mu \mathrm{m}$ & $120 \mu \mathrm{m}$ \\
III ${ }^{1}$ & $8 \mu \mathrm{m}$ & $400 \mu \mathrm{m}$ & $300 \mu \mathrm{m}$ \\
IV & $8 \mu \mathrm{m}$ & $300 \mu \mathrm{m}$ & $120 \mu \mathrm{m}$ \\
\hline
\end{tabular}

Table 1: Lithographic patterns

Improvements in the rate capability, which is essential for LHC applications, were realized with "low resistivity" substrates of $10^{-9} \Omega \mathrm{cm}$. Very promising are electronically conducting glasses [10], which, however, are difficult to produce as thin sheets. An alternative might be substrates where the conductivity is increased by ion bombardment [11] or by adding a very thin layer of conducting material like germanium on top of the structured substrate [12]. Rate capabilities of about $10^{7} / \mathrm{mm}^{2} / \mathrm{s}$ can be achieved with the "micro-gap chamber" where the gap between the anode and the cathode is only a few micrometers [13]

Another potentially interesting application for thin MSGCs are vertex jet chambers [14]. Here the particles would travel parallel to microstrip foils. The electrostatic problems of wire planes would be avoided. To obtain enough primary ionization a pitch of about $2 \mathrm{~mm}$ would be necessary. It is realizable with wide cathode strips which also improve the gain. With a slow gas like dimethylether 50 measurements of $40 \mu \mathrm{m}$ precision could be feasible for a path of $10 \mathrm{~cm}$ length only.

\section{The experimental conditions}

We investigated several structures varying in the width of the strips, the pitch, in the thickness of the substrate and in the material which was either a low resistivity glass (D263, DESAG, Gruenenplan, Germany) or polyimide (Kapton H). The glass substrates were $500 \mu \mathrm{m}$ and $100 \mu \mathrm{m}$ thick, the polyimide foils $125 \mu \mathrm{m}$ and $7 \mu \mathrm{m}$. The sensitive area was in all cases $3.5 \times 2 \mathrm{~cm}^{2}$. Table 1 summarizes the geometric parameters of the lithographic structure.

The $100 \mu \mathrm{m}$ glass plates have maximum tolerances of $\pm 15 \mu \mathrm{m}$ for sizes of $360 \mathrm{x}$ $415 \mathrm{~mm}^{2}$. We measured maximum deviations of $\pm 5 \mu \mathrm{m}$ for a 4 " $\times 4$ " sample. The glass sheets can be bent to cylinders with minimum diameters of about $7 \mathrm{~cm}$ before they break. These plates are very delicate to handle and require special care during the lithographic process, especially when large sensitive areas are needed. To spin-on the photoresist we constructed a special device which allowed to suck the glass to the rotating plate. The chromium electrodes were produced in a wet edging lithographic process by industry (Baumer IMT, Greifensee, Switzerland).

The Kapton foils were structured by a method, which combines a galvanic process with wet edging ('Galvadur' process, by Heidenhain, Traunreut, Germany). The electrodes were made out of gold. The quality of the lithography is excellent, similar to that on glass plates.

During the measurements the cathode strips were grounded, the anodes and the drift

\footnotetext{
${ }^{1}$ Mask lent to us by Dr. A. Oed.
} 
electrode were put to voltages of typically $-3000 \mathrm{~V}$ and $600 \mathrm{~V}$, respectively. The drift electrode is located $10 \mathrm{~mm}$ above the substrate. The back plane of all structures was put to ground if not stated differently. The back plane of the $7 \mu \mathrm{m}$ thin polyimide foil was left non conducting and the foil suspended freely in the gas. Simulations showed, and experiment confirmed, that the device cannot be operated at a fixed back plane potential, because, either the field lines from the anode or those from the drift electrode would end on the substate. Both cases have to be avoided.

The detectors were operated with the standard gas mixture of argon methane $90: 10$ and irradiated with a Fe-55 source with an intensity of up to about $10^{4}$ per $\mathrm{mm}^{2}$.

\section{Results}

In figure 1 we show the pulse height distributions for the glass and polyimide substrates. Except for the very thin Kapton foil, which was available only with a $300 \mu m$ distance between anode and cathode strips (geometry III) the geometry I was chosen. The slightly
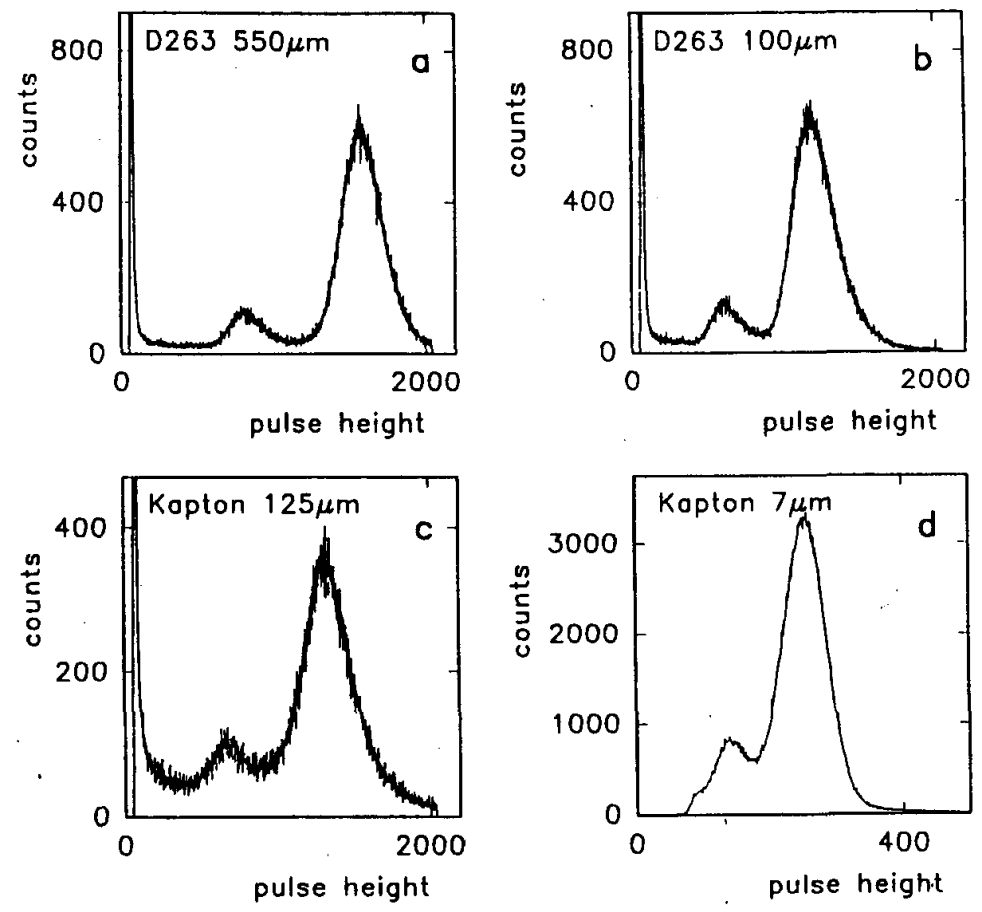

Figure 1: Pulse height distributions for a Fe-55 source. The structures a) to c) correspond to geometry I, $V_{A}=660 \mathrm{~V}, V_{D}=-3000 \mathrm{~V}$, the thin polyimide foil d) was structured according to geometry III, $V_{A}=920 \mathrm{~V}, V_{D}=-1500 \mathrm{~V}$.

worse resolution of the thin glass compared to the thick glass might be explained by thickness variations of the thin plate, which affect the electric field. The resolutions for the glass substrates are better than those for the Kapton foils. This might be due to the sensitivity of the plastic substrates to radiation. The rate was strongly varying 


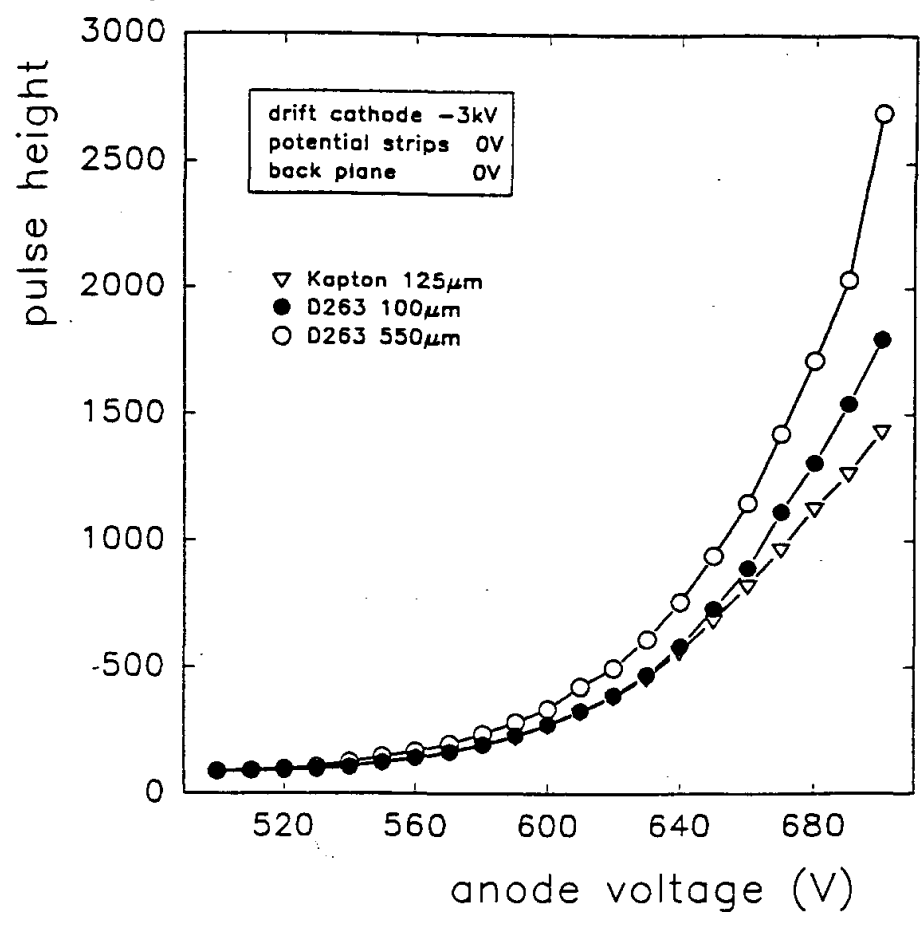

Figure 2: Gas amplification for glass plates and polyimide foil as a function of the anode voltage.

over the sensitive detector area. The gain for the $7 \mu m$ polyimide foil is smaller than for the thicker foils and the glass devices. This effect is qualitatively explained by the fact that for a very thin substrate without fixed back plane voltage the potential at the surface between the anode and the cathode strips is subdivided about linearly by the current flow. This leads to a low electric field at the anode. A $100 \mu \mathrm{m}$ glass plate with the same strip geometry as the $7 \mu \mathrm{m}$ polyimide foil showed a substantially higher gas amplification. The gain of the very thin Kapton foil was rather unstable. It increased during the first ten hours by about $30 \%$ and than started to decrease. This poor stability may be caused by radiation induced conductivity, which can depend on the field strength (for fields above $10 \mathrm{kV} / \mathrm{mm}$ ) and on the thickness [14]. Also charging up of the isolated back plane of the foil may contribute to gain fluctuations. Charges can be created by ionization and gas amplification in the gas behind the back plane. Gas amplification is possible until the field is reduced by deposited charges.

The gas amplification as a function of the anode voltage for geometry II for glass and the $125 \mu \mathrm{m}$ polyimide is displayed in figure 2 . The behavior of the three substrates are very similar. The gain is higher for the thick glass than for the thin glass and it is lowest for the Kapton foil.

For a given geometry of the structured substrate the gain decreases with increasing ratio $R_{\sigma}$ of surface to volume conductivity. The conductivities may depend on the field strength, on the irradiation, ion deposits, etc. and thus local variations are expected. For a qualitative discussion we only consider averaged values. The smaller gain for 


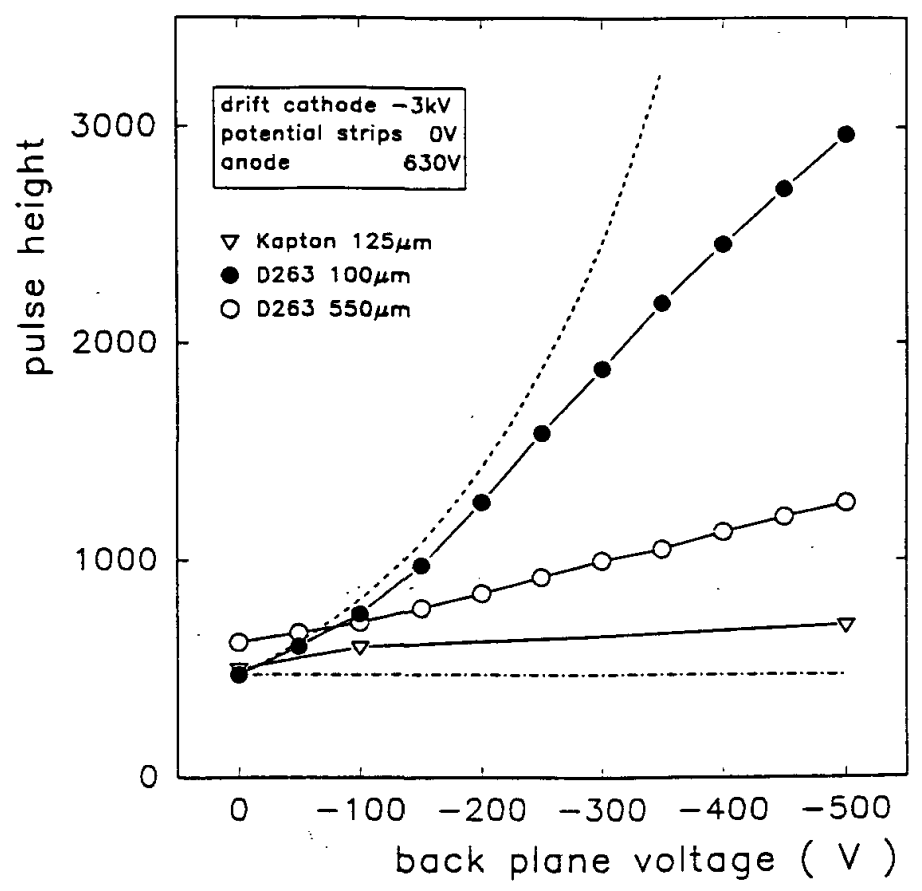

Figure 3: Gas amplification for glass plates and polyimide foil as a function of the back plane voltage.

polyimide relative to glass indicates that surface currents are more pronounced in the plastic material than in glass. A higher gain is expected for the thin glass plate relative to the thick plate, because the grounded back plane should increase the field near the anode. The opposite behavior is observed, probably due to a lower surface resistivity of the thin substrates (see below).

The measured dependence of the gain on the back plane voltage is shown in figure 3 together with the predictions for zero (dashed curve) and for infinite (dash-dotted curve) surface conductivities [15]. The computed curves are normalized to the measurement at zero back plane voltage. The measured curves lie between the extreme predictions. This indicates that both, surface and bulk conductivity contribute. As expected the influence of the back plane potential is weaker for the thick glass than for the thin glass. The curves of figure 3 again indicate a larger value of $R_{\sigma}$ for polyimide than for glass. A relatively large surface conductivity is needed to shield the field induced by the back plane electrode.

When the back plane voltage is decreased the gain immediately increases and then drops roughly exponentially to a stationary value. The time constants are a few minutes for the glass substrates but about ten times longer for polyimide.

The influence of the variation of the strip geometry on the gas amplification is shown in figure 4. Apart from the obvious dependence on the distance of the anode from the cathode strip, there is a sizable increase in gain with the width of the cathode strips. Especially for a jet chamber geometry, where tracks travel parallel to the structure, large 


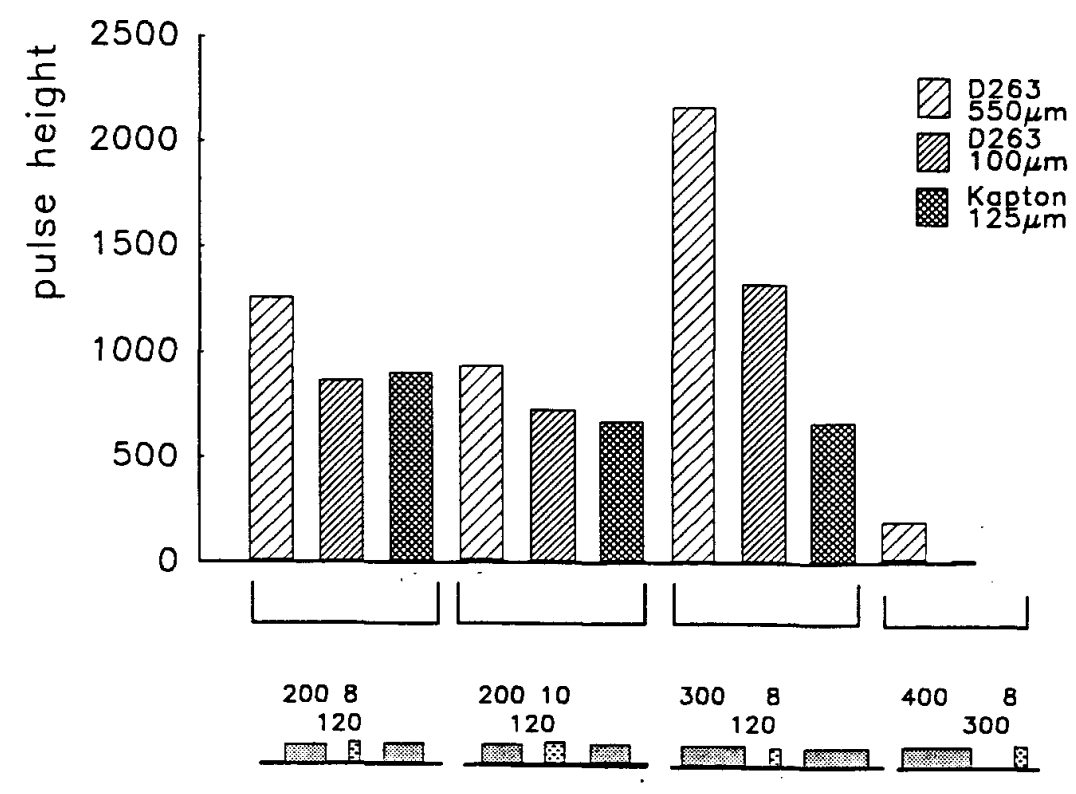

Figure 4: Comparison of gas gain for glass plates and polyimide foils with different lithographic patterns.

cathode strips are favorable for a large primary ionization and for the gain.

Also the responses to variations of the applied voltages and to changes in rate are different for glass and polyimide. Figure 5 shows the drop in gain with time when the voltage is applied. The substrates are irradiated with a collimated $5 \mathrm{mCi}$ Fe-55 source, leading to a rate of about $10^{4} / \mathrm{s} / \mathrm{mm}^{2}$. The lithographic pattern III was chosen, which has a wide gap between anode and cathode strips. The anode voltage was $700 \mathrm{~V}$ and the drift electrode was set to $-2000 \mathrm{~V}$.

The initial gain drop is faster for the glass than for the polyimide and faster for the thin glass than for the thicker plate. After the gain has settled to a stable value, the source was removed for two hours. The glass substrates are rather insensitive to the rate change, the Kapton structure however shows a dramatic drop in gain when the source is removed and recovers slowly when the irradiation starts again. At some occasions we had also observed a rise in gain for certain glass substrates, an indication of this behavior is visible for the thin glass.

The initial variation of the gain can qualitatively be explained by the charging of the surface by bulk and surface currents with a time constant increasing with the resistivity and with the distance of the back plane from the front surface of the substrate.

The drop in gain for the Kapton foil with the removal of the irradiation is interpreted by a rate depending ratio of surface to bulk resistivity. The radiation from the Fe55 source decreases the bulk resistivity. We have indeed measured a difference of the conductivities by roughly a factor two to three with the source on and off at voltages where no gas amplification occurs. Radiation induced conductivity in Kapton has been reported in literature [14]. Clearly charging up of the surface due to deposited positive ions cannot explain the rising gain for the polyimide structure.

The conductivity of glass shows little sensitivity to irradiation, however, only small changes are required to explain the effect of figure 5. With a different D263 sample 

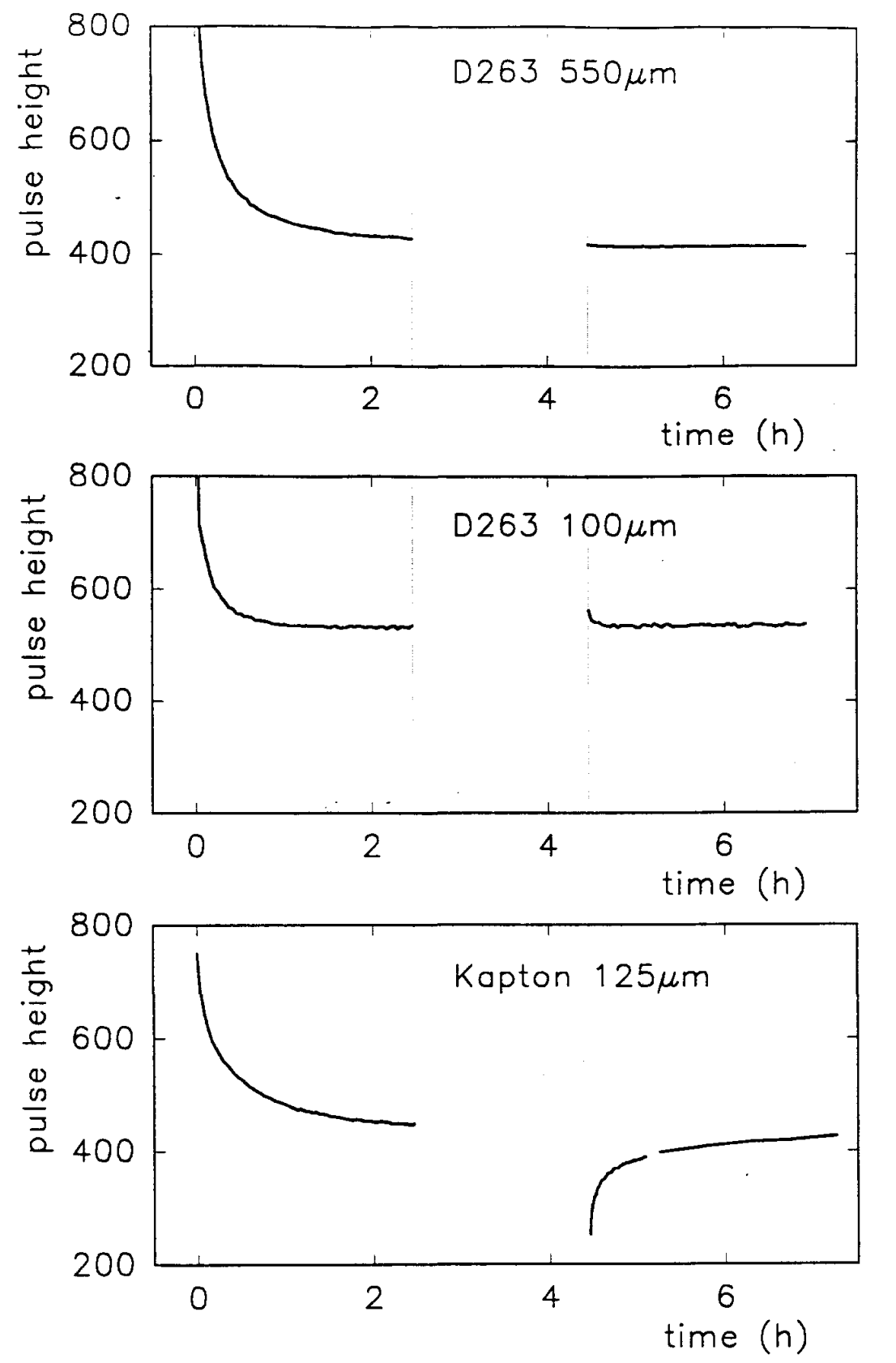

Figure 5: Rate dependence of gas gain for glass plates and polyimide foil. At $\mathrm{t}=0$ the high voltage was switched on, after 150 minutes the source was removed for two hours. 
we have observed a stronger $(15 \%)$ decrease with rate under similar conditions. The behavior of a substrate depends strongly on the surface conductivity which is hard to control.

At constant conditions and moderate rate $\left(<10^{4} / \mathrm{s} / \mathrm{mm}\right)$ no gain drift is observed for glass. We recorded small fluctuations of a few percent over some days which can be attributed to changes in atmospheric pressure.

We have also investigated gain variations with temperature for glass substrates.

The resistivity of glass follows to a very good approximation the expected behavior $\rho=A \exp (B / T)$. Our measurements in the range from $20^{\circ} \mathrm{C}$ to $100^{\circ} \mathrm{C}$ yielded a slope $\mathrm{B}=1.1 \times 10^{4}$ for $\mathrm{T}$ measured in $K$ and $\rho$ in $\Omega$. A temperature change by only one degree changes the resistivity by $14 \%$, or an increase of the temperature by $20^{\circ} \mathrm{C}$ increases the conductivity by an order of magnitude. The specific bulk resistivity at $25^{\circ} \mathrm{C}$ was $3.1 \times 10^{12} \Omega \mathrm{cm}$, identical for the thick and the thin glass.

The glass was heated in the gas enclosure to $45^{\circ} \mathrm{C}$. Then the heating was switched off and the gain was recorded during cooling down to $25^{\circ} \mathrm{C}$. It dropped steadily to a final value which was two third of the initial gain. The experiment was repeated for various voltage settings with similar results: An increase of the temperature of one degree increases the gain by $3 \%$ at room temperature. This means that not only the density of the gas, but also the temperature of the glass substrate has to be kept stable during the operation of MSGCs.

\section{Summary and conclusions}

We have tested thin glass plates of $100 \mu m$ thickness for their potential use as substrates for MSGCs. The results were very encouraging, no essential difference in behavior compared to the thicker plates was observed except for a somewhat higher conductivity. Compared to polyimide foils they are less sensitive to rate variations. We are currently producing structures on $50 \mu \mathrm{m}$ glass foils which can be bent to cylinders of $3 \mathrm{~cm}$ radius. In future we intend to try also $30 \mu m$ glass foils.

Very thin foils cannot be operated with conducting back planes and they have to be stretched in the gas volume. As a consequence a strong electric field will exist also in the gas volume at the back of the structure. Uncontrolled processes in this volume could be avoided by repeating the strip pattern of the front plane at the back plane. However, this would introduce additional complications in the lithographic artwork.

The glass plates showed sizable gain variations with temperature, which have to be taken care of.

Polyimide is not an ideal substrate for MSGCs. The high electric resistivity and its dependence on radiation lead to rather large variations with long time constants when the conditions change. Furthermore a single spark can destroy the whole structure. We intend to test in future polyimide with conductivity increased by ion bombardment (provided by V. Grishin, Lebedev Institute, Moscow).

The influence of the gas amplification on the strip widths and distances qualitatively corresponds to the naive expectations, except for a rather large increase in gain with the increased cathode width. 


\section{References}

[1] A. Oed, Nucl. Instr. and Meth. A263 (1988) 351.

[2] F. Angelini et al., Nucl. Instr. and Meth. A283 (1989) 755.

[3] F. Hartjes et al., Nucl. Instr. and Meth. A289 (1990) 384.

[4] F. Angelini et al., Nucl. Phys. 23A (1991) 254.

[5] F. Angelini et al., CERN-PPE 91-122 (1991).

[6] C. Budtz-Jorgenson et al., Rev. Sci. Instr. 63(1) (1992) 648.

[7] F. Angelini et al., Nucl. Instr. and Meth. A314 (1992) 450.

[8] H. Stahl et al., Nucl. Instr. and Meth. A297 (1990) 95.

[9] R. Bouclier et al., IEEE Trans. Nucl. Sci. NS-39 (1992) 650.

[10] R. Bouclier et al., Nucl. Instr. and Meth. A323 (1992) 240.

[11] R. Bouclier et al., Nucl. Instr. and Meth. A332 (1993) 100.

[12] M. Heidrich, Contribution to Workshop on Progress in Gaseous Microstrip Proportional Counters, Grenoble 1993, unpublished.

[13] F. Angelini et al., Pisa preprint, INFN PI/AE 93/10 (1993).

[14] A.H. Walenta, private communication.

[15] R. Filho et al., IEEE Trans. on Electr. Insul. EI-21 (1986) 431.

[16] S. Schmidt et al., Siegen preprint, in preparation. 